\title{
Modelling the impacts of seasonal drought on herbage growth under climate change
}

Pierluigi Calanca

LiveM 2016, 15-16 June 2016, Potsdam 


\section{$\oplus$ Drought and growth}
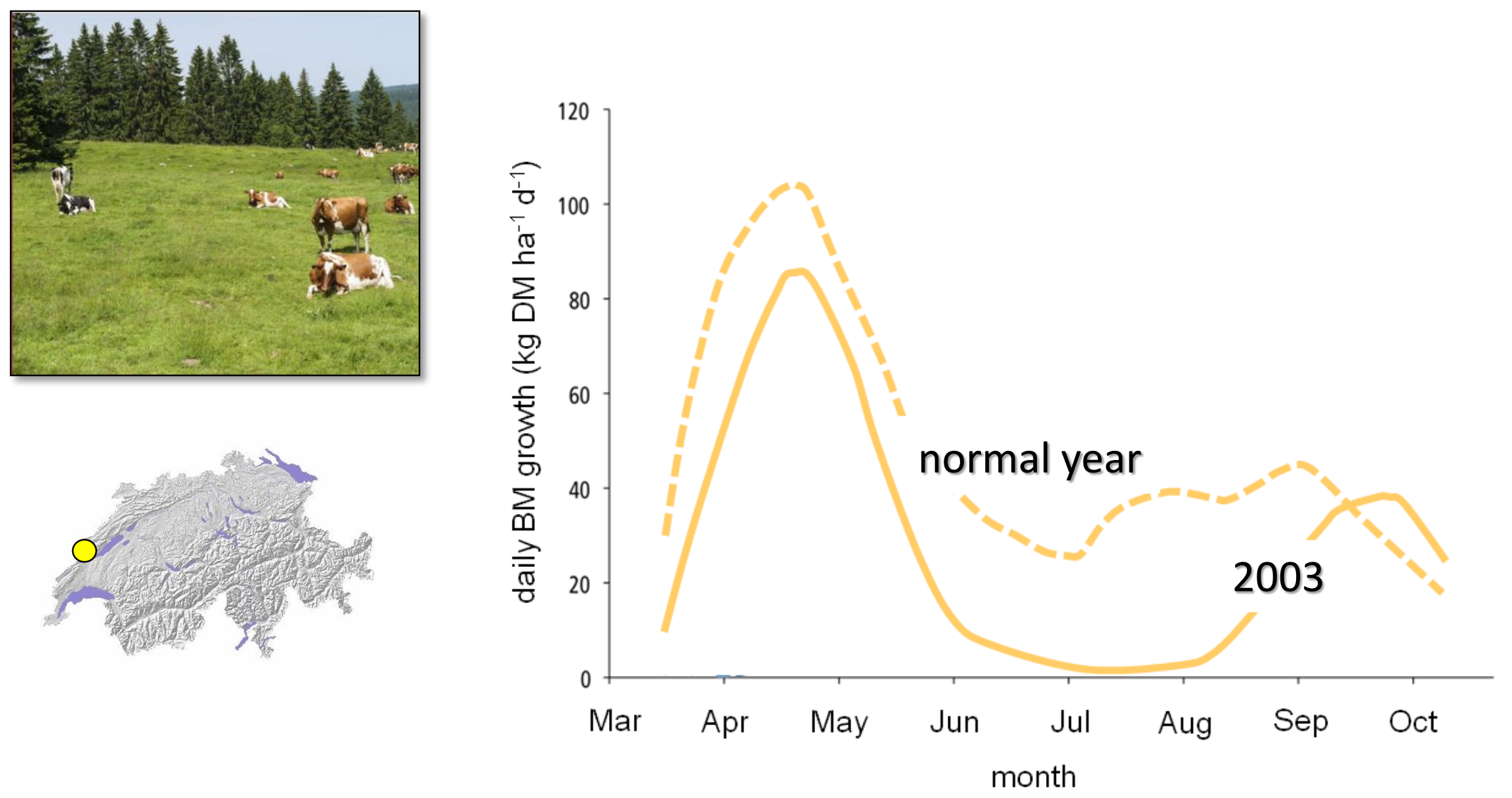

Mosimann et al. (2013) 


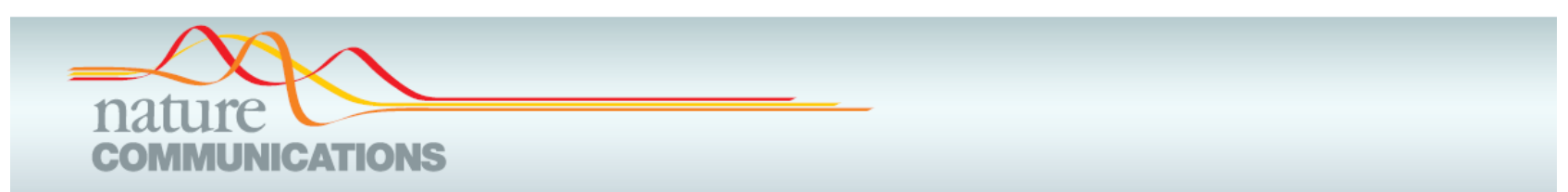

\section{ARTICLE}

Received 30 Oct 2014 | Accepted 10 Apr 2015 | Published 14 May 2015 DOl: 10.1038/ncomms8148 $\quad$ OPEN

\section{Long-term decline in grassland productivity driven by increasing dryness}

E. N. J. Brookshire ${ }^{1} \&$ T. Weaver ${ }^{2}$

a

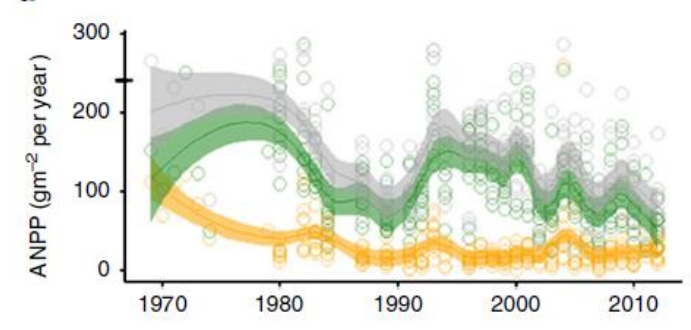

b

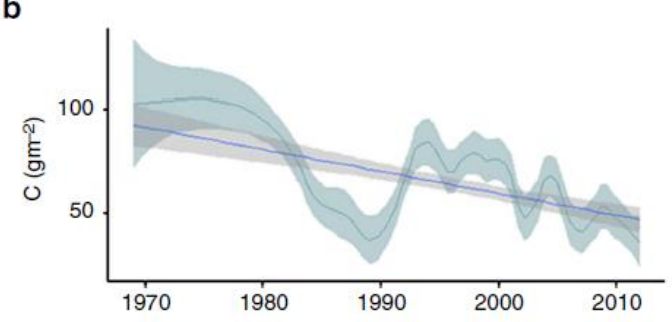

C
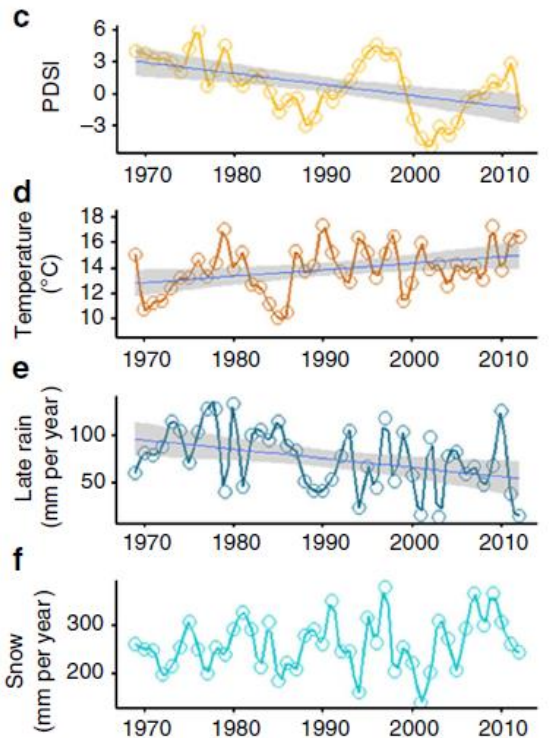

Figure 1 | Long-term pattern of grassland production and climate variation. (a) Above-ground net primary production in control plots showing all plot data (points) and locally weighted trend lines with $95 \%$ confidence bands for total (grey), grass (green) and forb (orange) production. The horizontal black bar on the $y$ axis is ANPP for 1965-1967 at a nearby subalpine grassland (ref. 20). (b) Change in total above-ground carbon pools with $95 \%$ confidence bands. (c) Time series of the regional Palmer Drought Severity Index (PDSI, lower values indicate increasing dryness), (d) September temperature, (e) latesummer rainfall and $(\mathbf{f})$ annual snowfall. All significant $(P<0.001)$ trends in $\mathbf{b}-\mathbf{f}$ are shown with a regression line (blue) and $95 \%$ confidence intervals. 


\section{$\oplus$ The future}

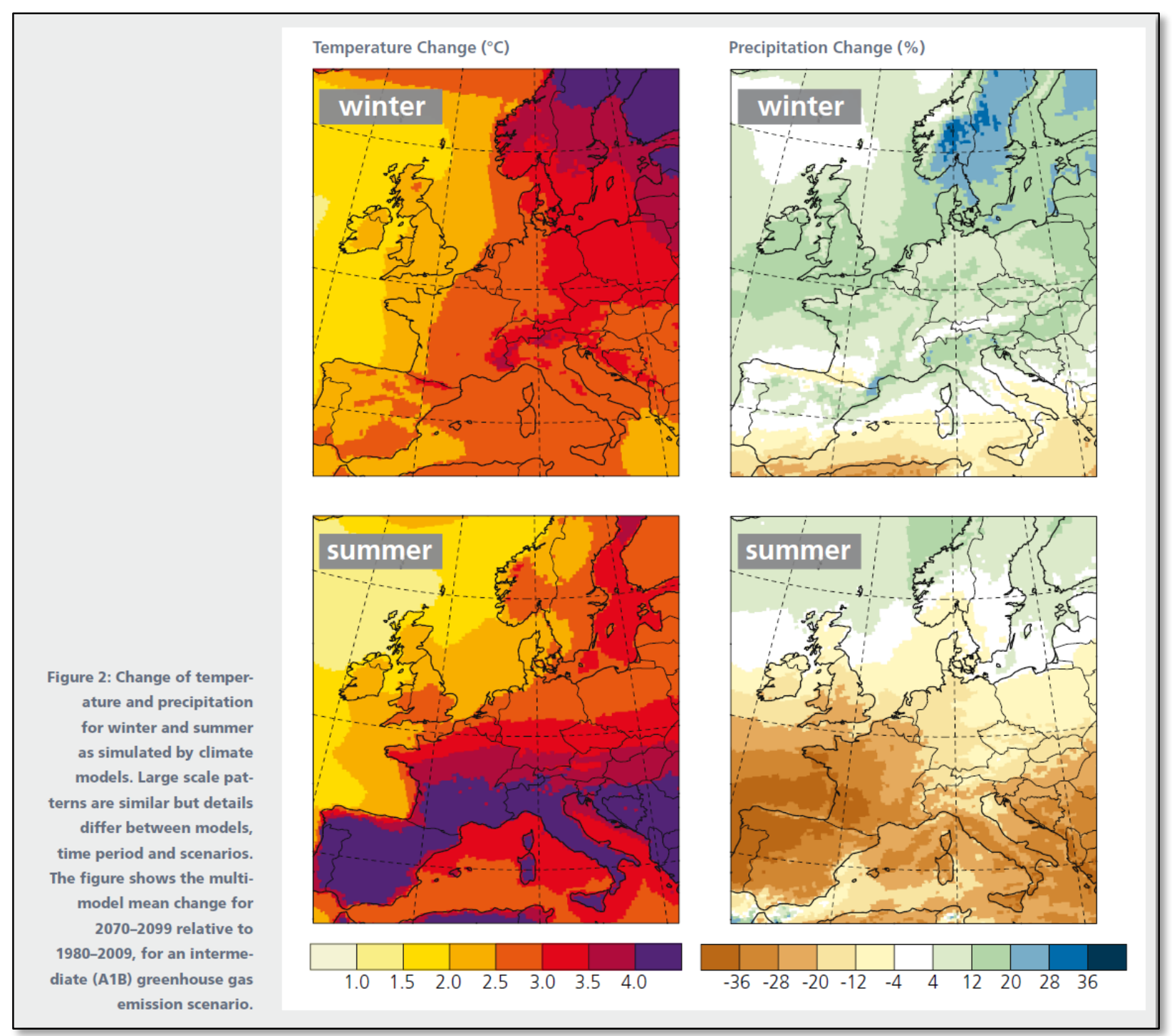

CH2O11 (2011) 
$\uplus$ Direct effects of water deficit on plant physiology

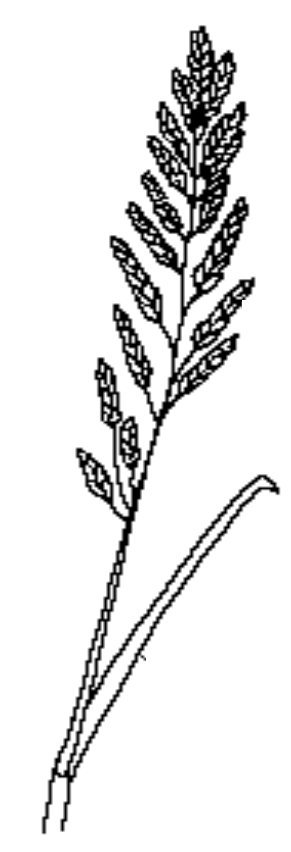

- Photosynthesis \& assimilation $\downarrow$

- LAl, biomass $\downarrow$

- SLA $\downarrow$, leaf dry matter content $\uparrow$

- Leaf lifetime $\downarrow$

- Root machinery $\uparrow$

- BNF $\downarrow$

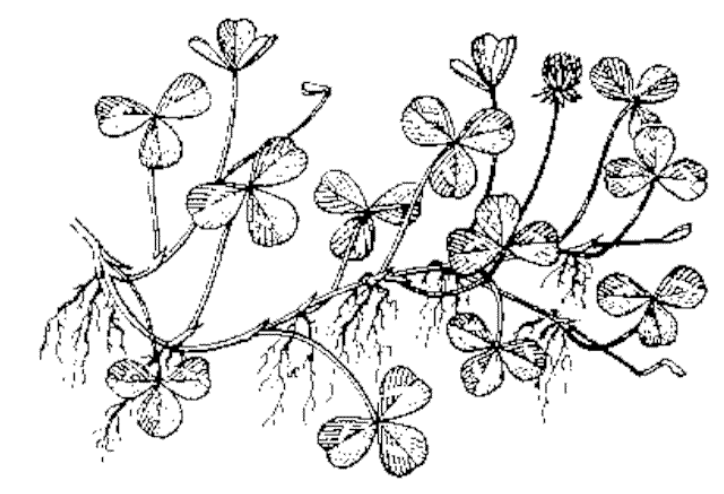


$\oplus$ Other important effects

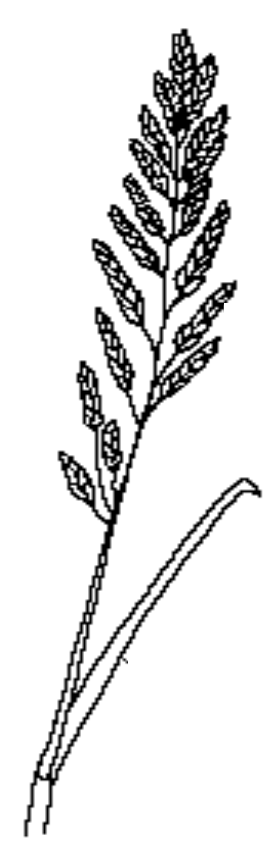

- Increasing temperature

$\Rightarrow$ growing season $\uparrow$

- Elevated $\mathrm{CO}_{2}$ concentrations

$\Rightarrow$ water \& $\mathrm{N}$ use efficiency $\uparrow$

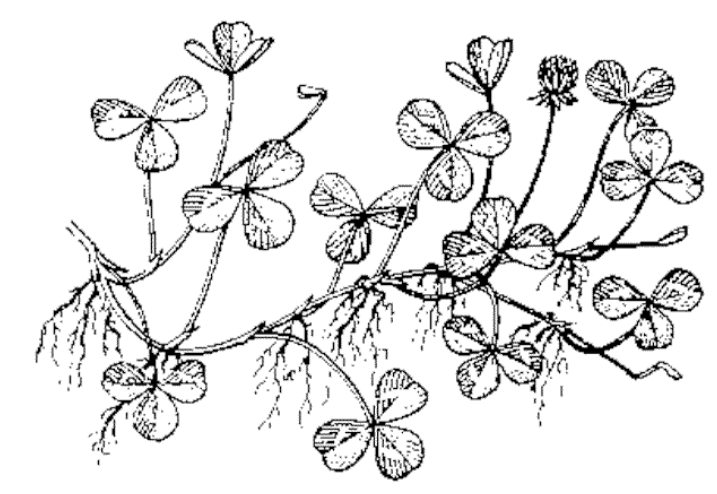

Meisser (2013) 
\# Grassland models

Model complexity

low

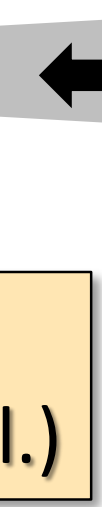

gap

high
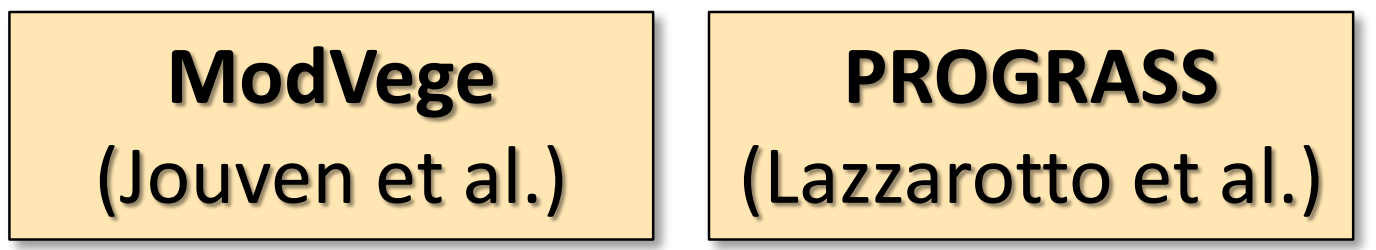

ecosys (Grant)

\section{PaSisso \\ (Riedo et al.)}

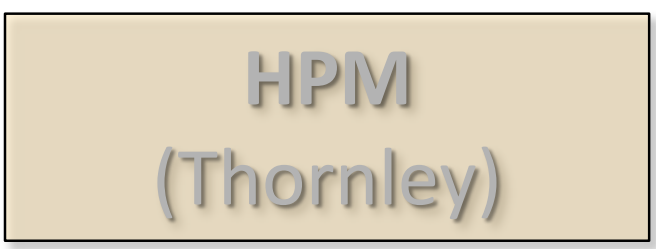




\section{$\oplus$ Grassland models: ecosys}
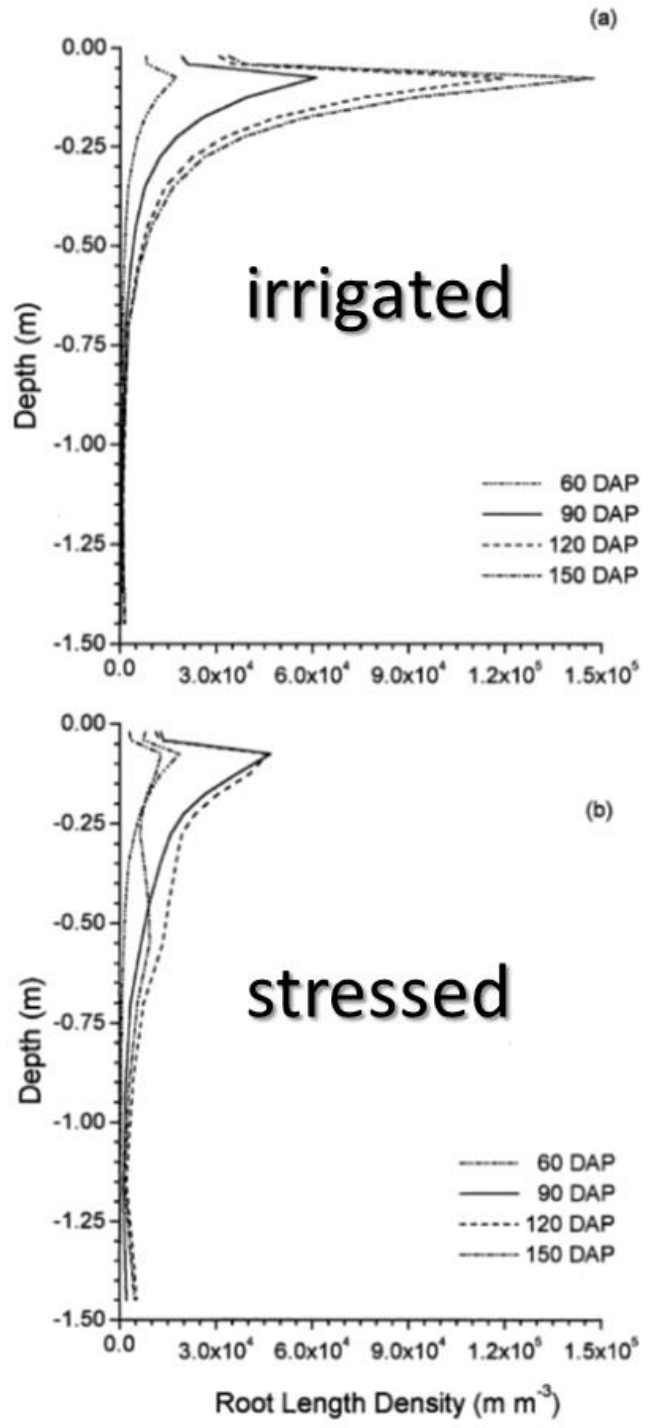

Fig. 5. Vertical profiles of root length density simulated $30,60,90,120$ and 150 days after planting (DAP) under $30 \mathrm{~g} \mathrm{~N} \mathrm{~m}^{-2}$ of fertilization and (a) $50 \mathrm{~mm}$ week $^{-1}$ of irrigation, or (b) zero irrigation. 


\section{$\oplus$ Model performance: ModVege}

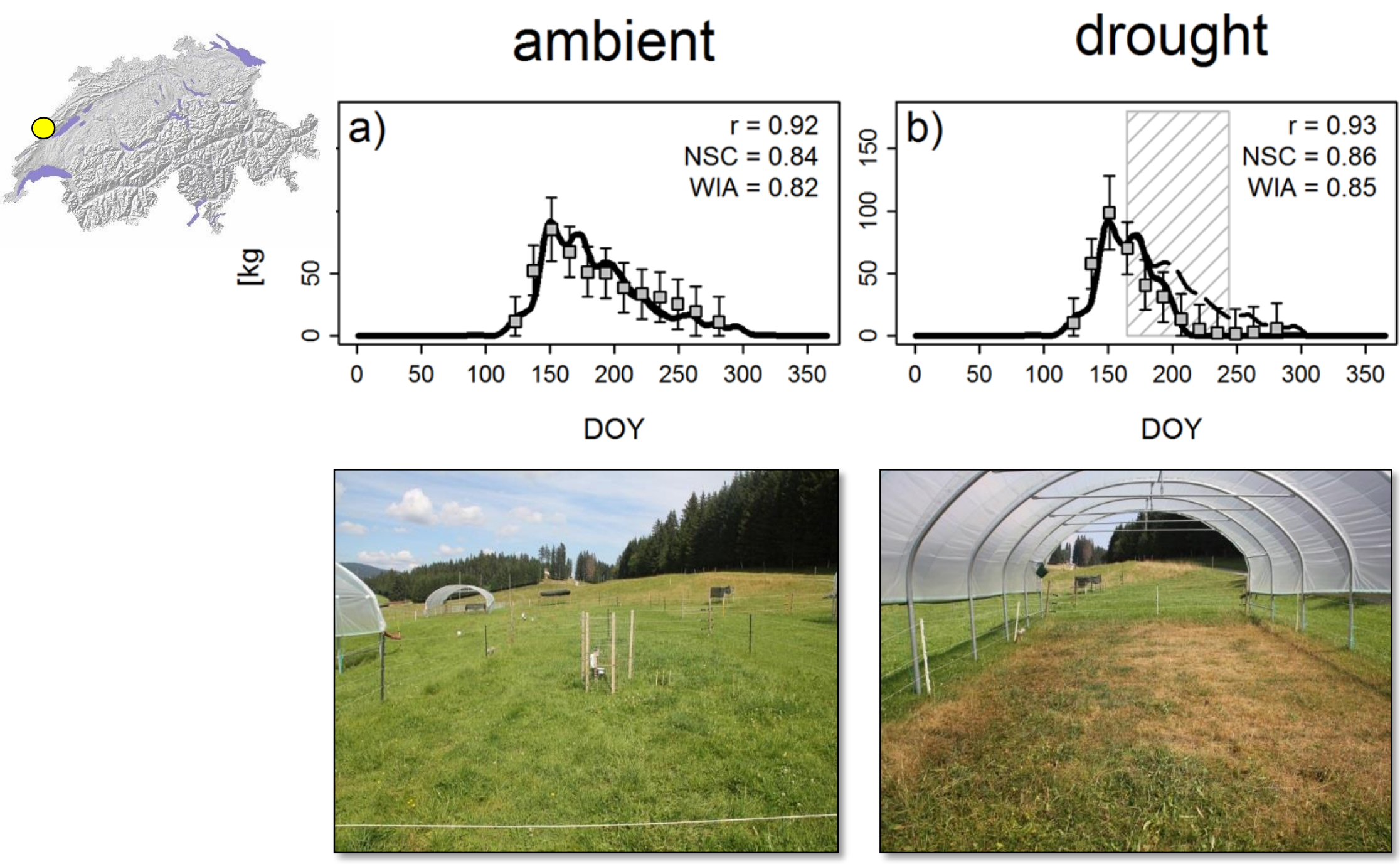

Calanca et al. (2016) 


\section{Model performance}

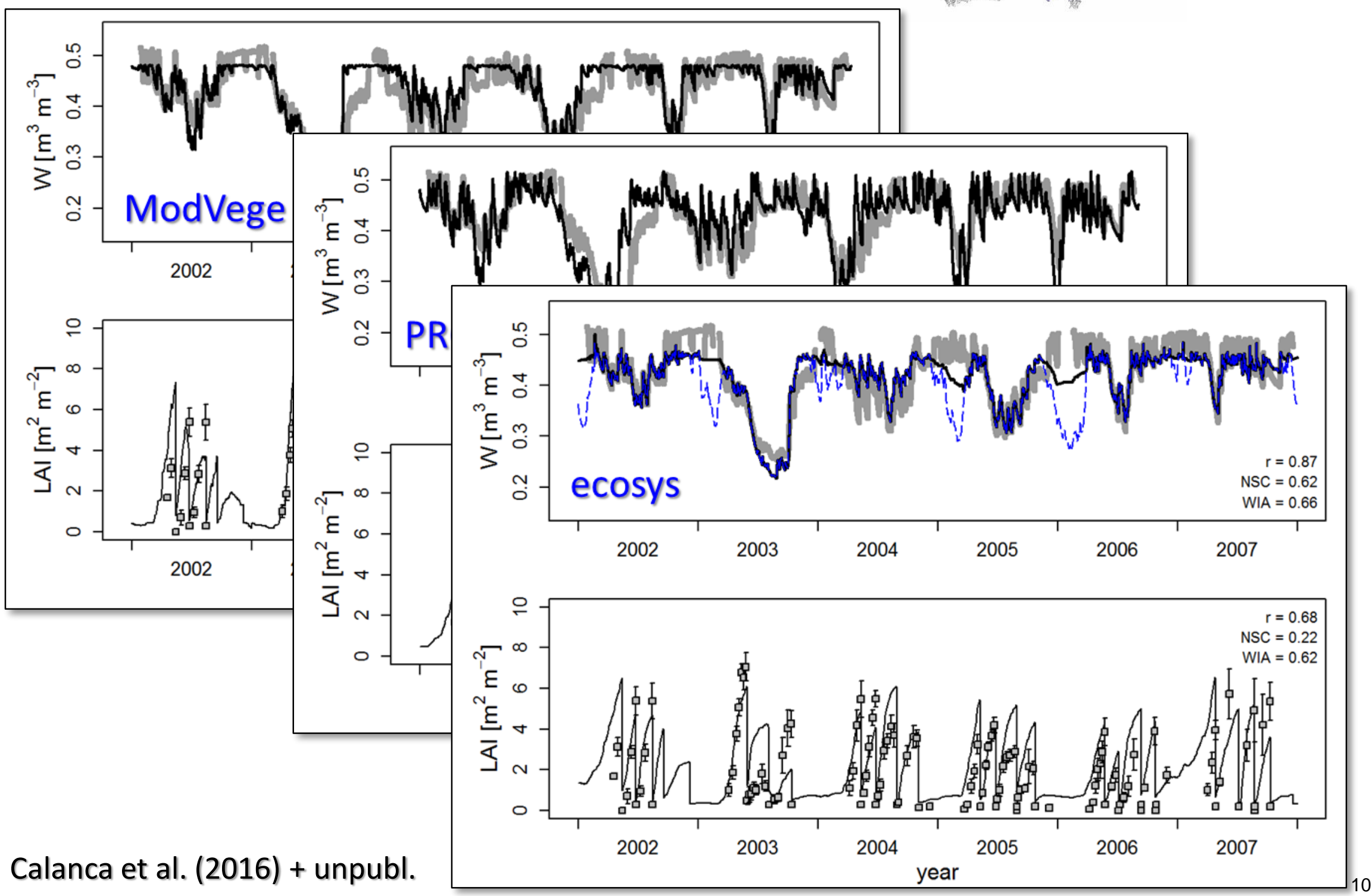




\section{\# Model performance}
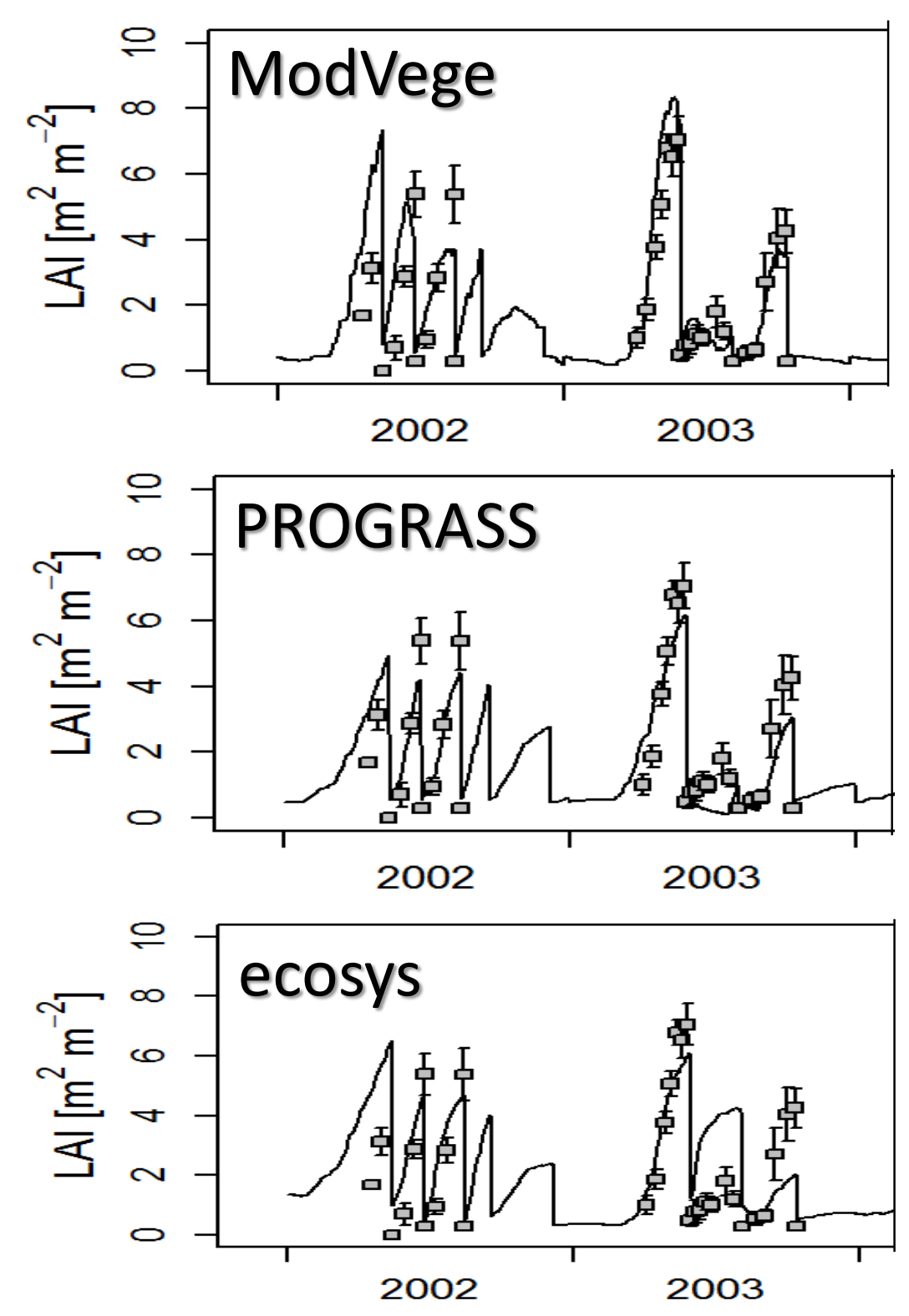


\section{$\uplus \quad$ Model application: ModVege}

Changins
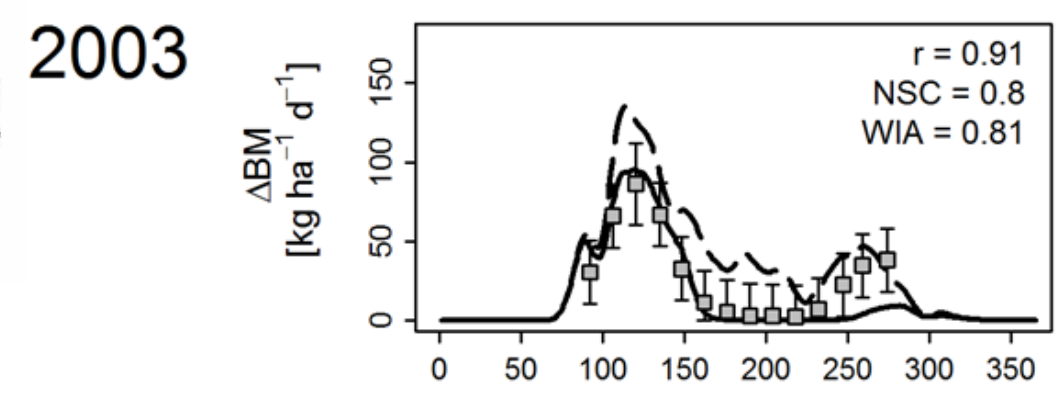

2004
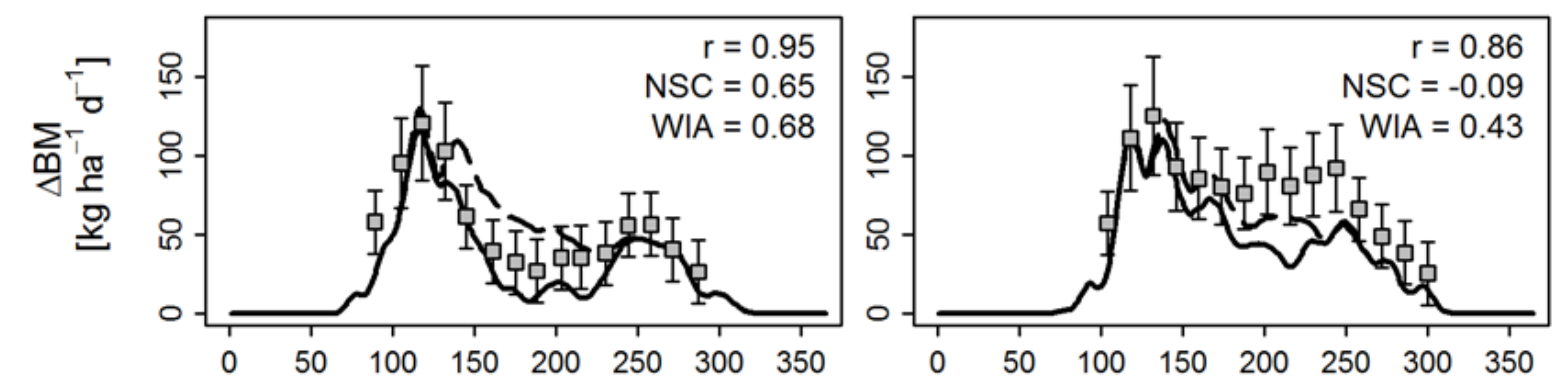

2006

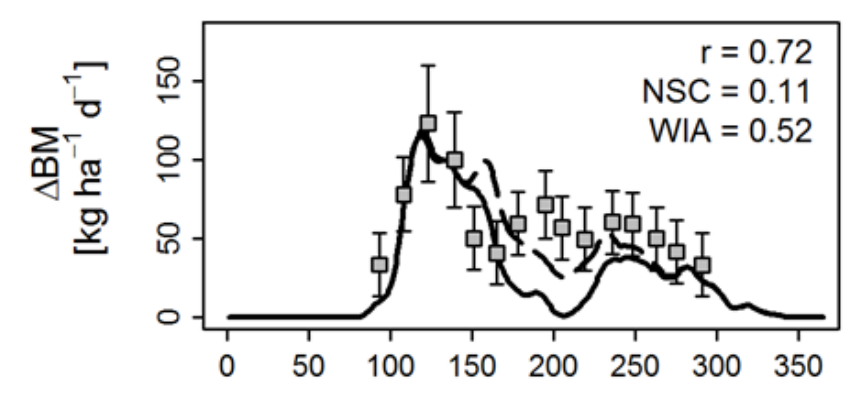

Posieux
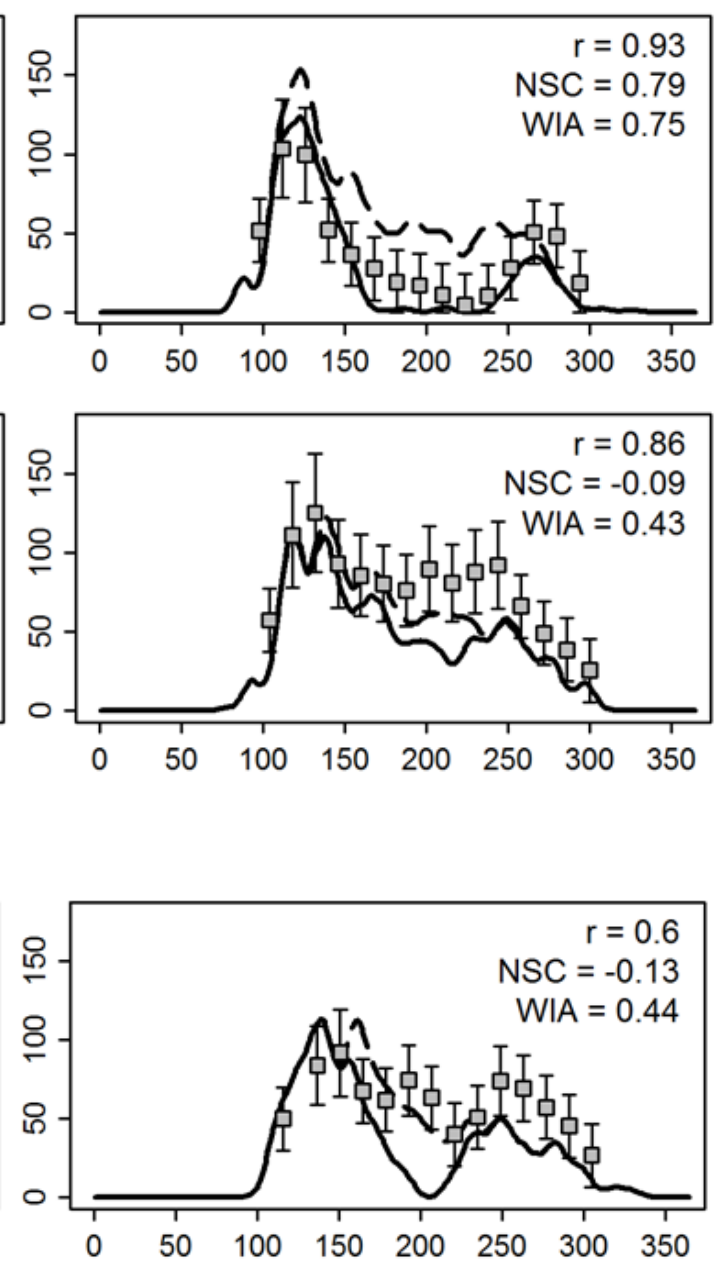

Calanca et al. (2016) 


\section{$\Psi$ Model application: ModVege}

Changins

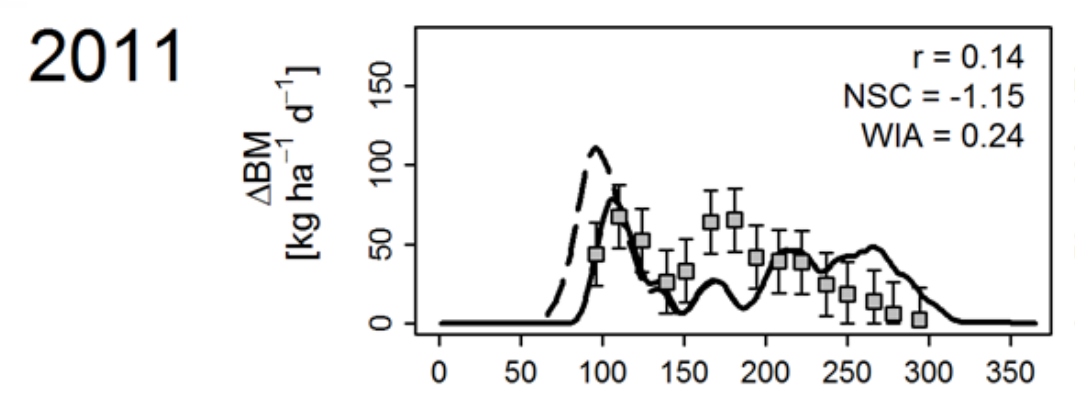

2012

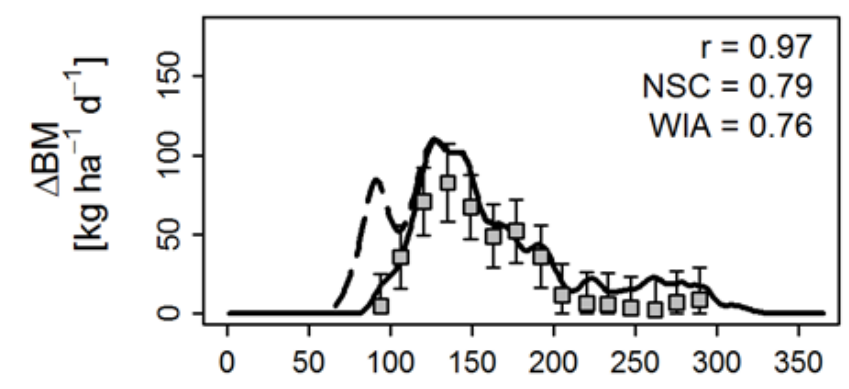

Posieux
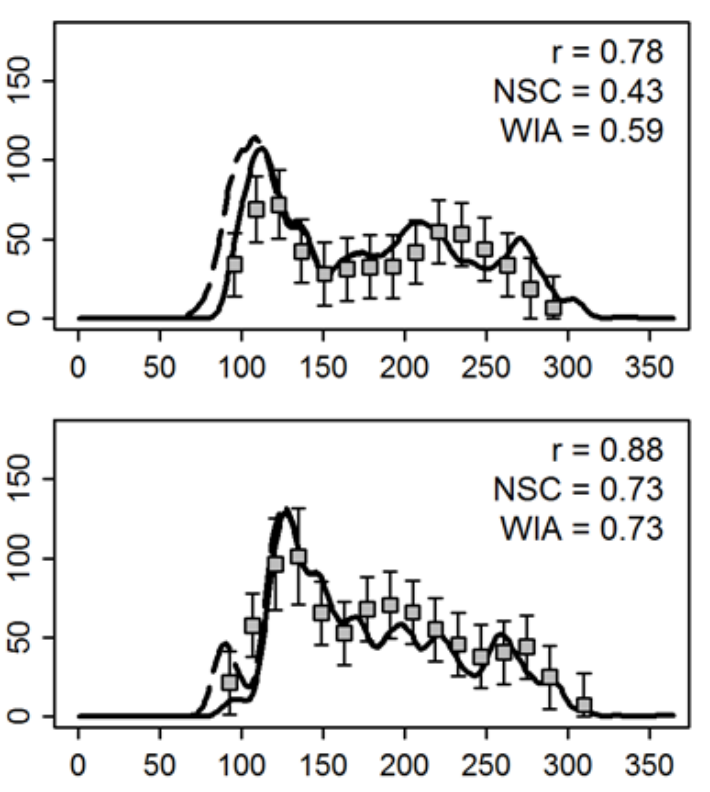


\section{$\oplus$ Root dynamics}
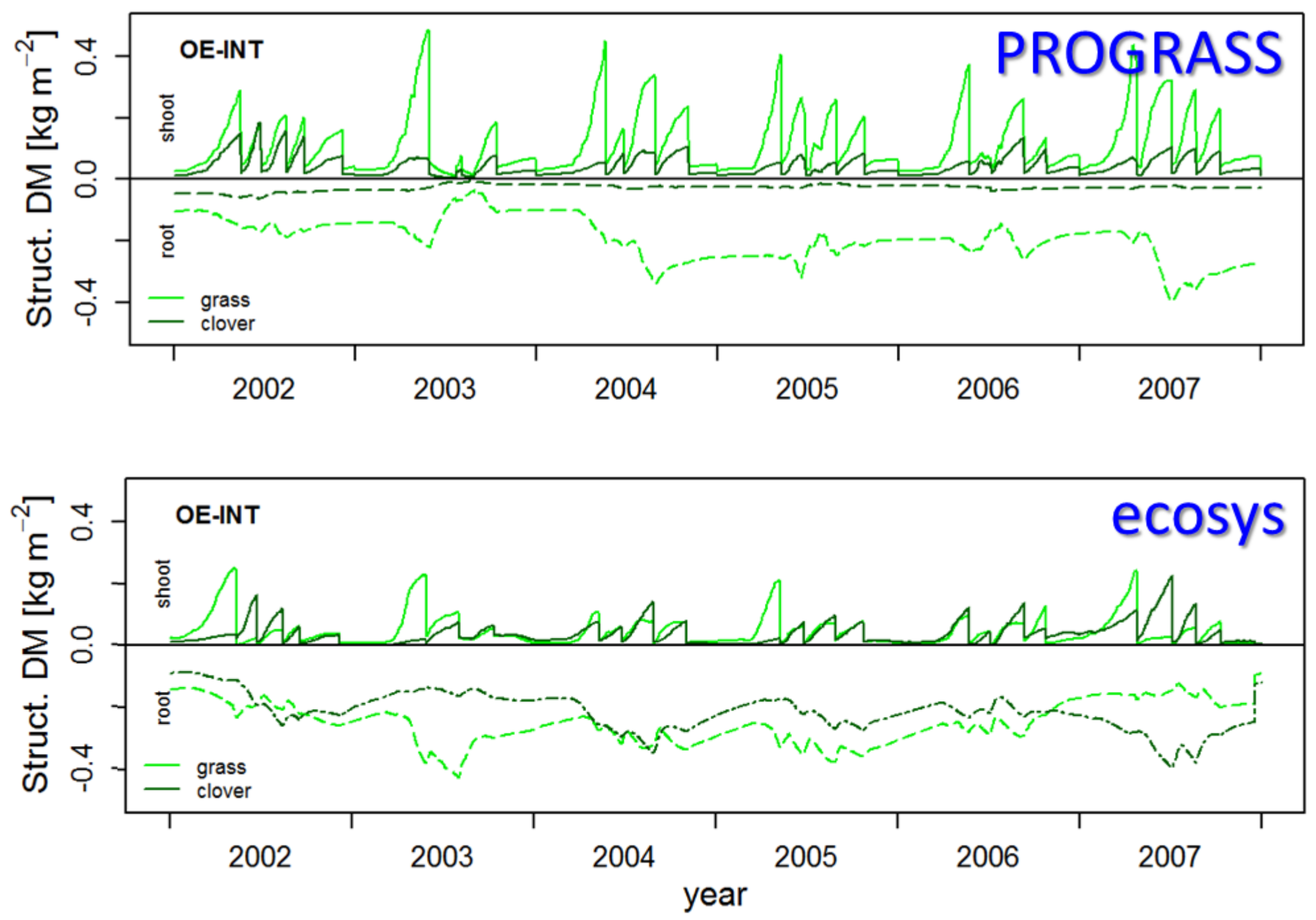
$\oplus$ Co-existence \& interactions
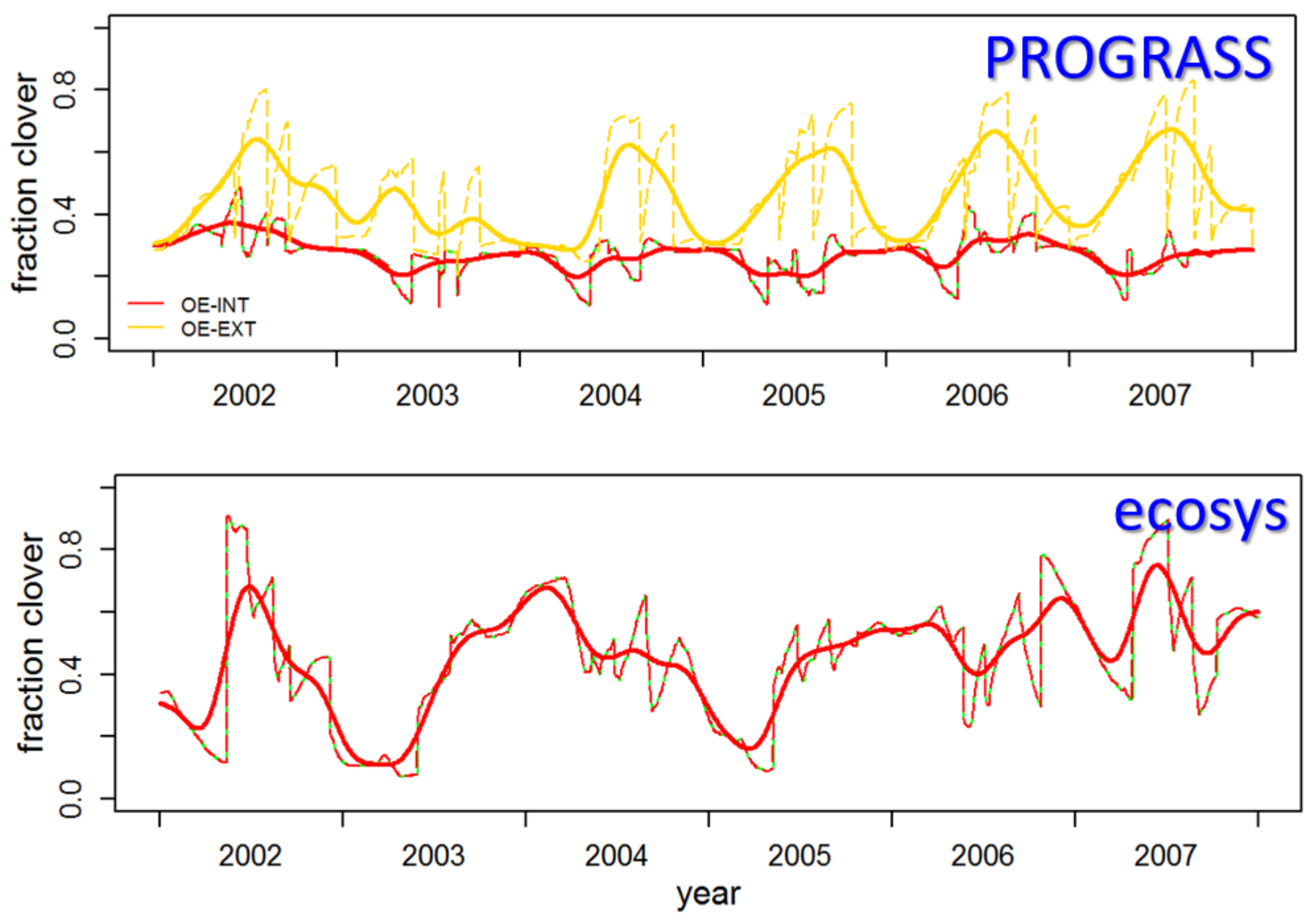
$\oplus$ Conclusions

The sensitivity of grasslands with respect to drought depends on

- Phenology, overwintering \& winter mortality

- Root dynamics

- Community dynamics \& species interactions

- Short-term effects of management

- Long-term effects of management

There is room for improving the formulation of these processes in current grassland models

\section{Moreover ...}




\section{* Complicating factors}
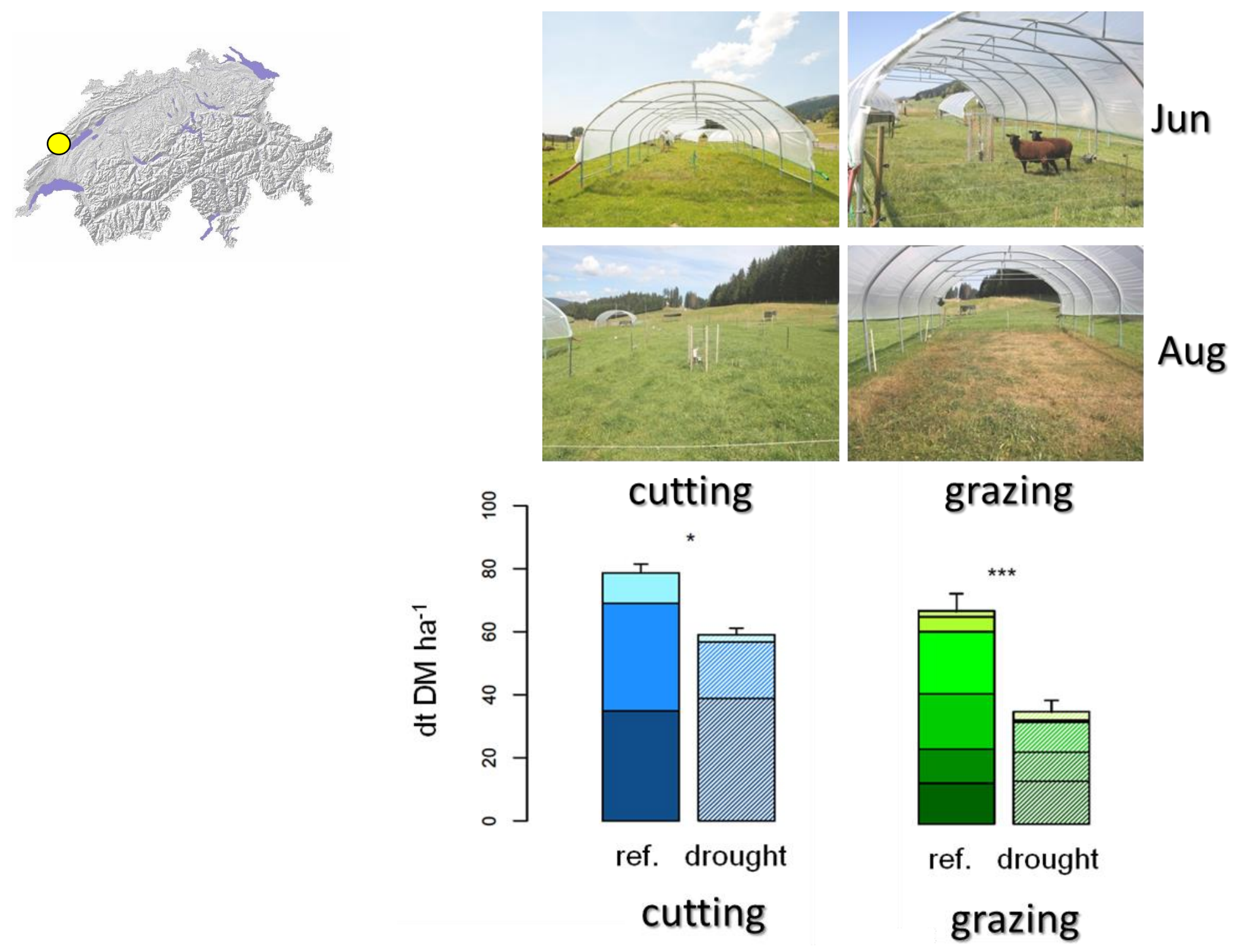

Meisser et al. (2013) 


\begin{tabular}{|ll|}
\hline $\begin{array}{l}\text { Journal of } \\
\text { Ecology } 2006\end{array}$ & ESSAY REVIEW \\
94, 801-814 & The Park Grass Experiment 1856-2006: \\
& its contribution to ecology \\
& JONATHAN SILVERTOWN, PAUL POULTON*, EDWARD JOHNSTON*, \\
& GRANT EDWARDS + MATTHEW HEARD ${ }^{*}$ and PAMELA M. BISS \\
\hline
\end{tabular}
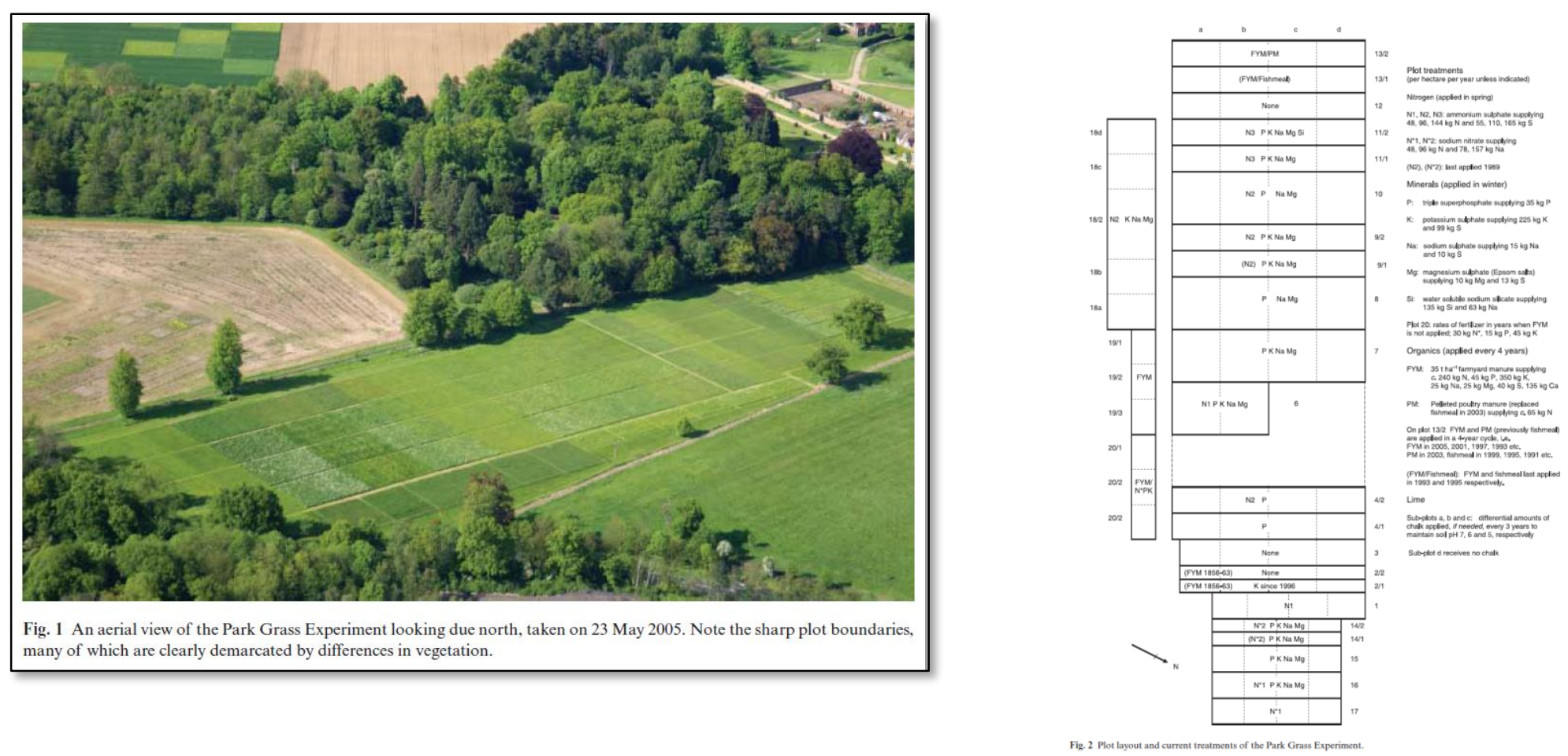


\section{Complicating factors}

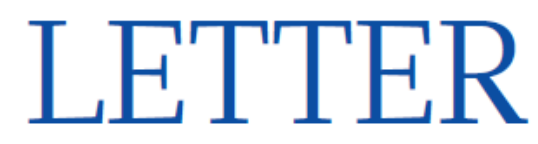

Grassland biodiversity bounces back from long-term nitrogen addition

J. Storkey ${ }^{1}$, A. J. Macdonald ${ }^{1}$, P. R. Poulton ${ }^{1}$, T. Scott ${ }^{1}$, I. H. Köhler ${ }^{2} \dagger$, H. Schnyder ${ }^{2}$, K. W. T. Goulding ${ }^{1} \&$ M. J. Crawley ${ }^{3}$

PROCEEDINGS

THE ROYAL
SOCIETY
Proc. R. Soc. B (2006) 273, 1149-1154 doi: $10.1098 / \mathrm{rspb} .2005 .3428$ Published online 24 January 2006

Soil moisture mediates association between the winter North Atlantic Oscillation and summer growth in the Park Grass Experiment

P. S. Kettlewell ${ }^{1, *}$, J. Easey ${ }^{1}$, D. B. Stephenson ${ }^{2}$ and P. R. Poulton ${ }^{3}$ 